Int. J. Contemp. Math. Sci., Vol. 1, 2006, no. 9, 417-418

\title{
Rational curves in grassmannians and their Plücker embeddings: an application
}

\author{
E. Ballico, ${ }^{1}$ S. Pasotti ${ }^{2}$ and F. Prantil ${ }^{3}$ \\ Dept. of Mathematics, University of Trento \\ 38050 Povo (TN), Italy \\ ballico@science.unitn.it \\ pasotti@science.unitn.it \\ prantil@science.unitn.it
}

\begin{abstract}
Here we prove a corollary of [1, Thm. 1], providing sufficient condition for the existence of $\alpha$-stable coherent systems of type $(n, d, k)$ for some $k>n$.
\end{abstract}

\section{Mathematics Subject Classification: $14 \mathrm{H} 60$}

Keywords: stable vector bundles on curves; coherent system; Grassmannian; spanned vector bundle

\section{INTRODUCTION}

Let $X$ be a smooth and connected projective curve. A coherent system on $X$ is a pair $(E, V)$ such that $E$ is a vector bundle on $X$ and $V \subseteq H^{0}(X, E)$ is a linear subspace. The pair $(E, V)$ is of type $(n, d, k)$ if $\operatorname{rank}(E)=n, \operatorname{deg}(E)=d$ and $\operatorname{dim}(V)=k$. Fix $\alpha \in \mathbb{R}$. Let $\mu(E):=d / n$ denote the slope of $E$. Set $\mu_{\alpha}(E, V):=\mu(E)+\alpha k / n$. The real number $\mu_{\alpha}$ is called the $\alpha$-slope of the pair $(E, V)$. A coherent subsystem $(F, W) \subseteq(E, V)$ is a coherent system such that $F \subseteq E$ and $W \subseteq V \cap H^{0}(X, F)$. The pair $(E, V)$ is said to be $\alpha$-stable (resp. $\alpha$-semistable) if $\mu_{\alpha}(F, W)<\mu_{\alpha}(E, V)$ (resp. $\left.\mu_{\alpha}(F, W) \leq \mu_{\alpha}(E, V)\right)$ for all proper coherent subsystems $(F, W)$ of $(E, V)$. For the general theory of coherent systems and several results on the moduli schemes of $\alpha$-stables coherent systems see [7], [4], [2], [5], [6] and [3]. Here we prove a corollary of [1, Thm. 1], providing sufficient conditions for the existence of $\alpha$-stable coherent systems of type $(n, d, k)$ for some $k>n$.

Proposition 1. Fix $\alpha \in \mathbb{R}$ and integers integers $n \geq 2, a_{1} \geq \cdots \geq a_{n}>0$ and $k$ such that $\left(\begin{array}{l}k \\ n\end{array}\right) \leq 1+n a_{n}$ and $\alpha>\left(n a_{1}-\sum_{i=1}^{n} a_{i}\right) /(\bar{k}-n)$. Set $E:=\oplus_{i=1}^{n} \mathcal{O}_{\mathbf{P}^{1}}\left(a_{i}\right)$ and take a general $k$-dimensional linear subspace $V$ of $H^{0}\left(\mathbf{P}^{1}, E\right)$. Then the coherent system $(E, V)$ is $\alpha$-stable. Furthermore, for all coherent subsystems $(F, W)$ of $(E, V)$

\footnotetext{
${ }^{1}$ The author was partially supported by MIUR and GNSAGA of INdAM (Italy).

${ }^{2}$ The author was partially supported by MIUR and GNSAGA of INdAM (Italy) and HPMTCT-2001-00277.

${ }^{3}$ The author was partially supported by MIUR and GNSAGA of INdAM (Italy) and HPMTCT-2001-00277.
} 
such that $1 \leq \operatorname{rank}(F)<n$ we have $\mu_{\alpha}(E, V)-\mu_{\alpha}(F, W) \geq\left(\sum_{i=1}^{n} a_{i}\right) / n+(k-$ n) $\alpha / n-a_{n}$

Proof. By [1] for all integers $r$ such that $1 \leq r<n$ and all rank $r$ subsheaves $F$ of $E$ we have $\left.\operatorname{dim}(V) \cap H^{0}\left(\mathbf{P}^{1}, F\right)\right) \leq r$. Since $\mu_{+}(E)=a_{1}$, we have $\mu(F) \leq a_{1}$. Thus $\mu_{\alpha}(F, W) \leq a_{1}+\alpha<\left(\sum_{i=1}^{n} a_{i}\right) / n+(k / n) \alpha$, concluding the proof.

\section{REFERENCES}

[1] E. Ballico, Rational curves in Grassmannians and their Plücker embeddings, preprint.

[2] S. B. Bradlow, O. García-Prada, V. Muñoz and P. E. Newstead, Coherent systems and BrillNoether theory, Internat. J. Math. 14 (2003), no. 7, 683-733.

[3] L. Brambila-Paz, Non-emptiness of moduli spaces of coherent systems, e-preprint arXiv:math.AG/0412285.

[4] A. King and P. E. Newstead, Moduli of Brill-Noether pairs on algebraic curves, Internat. J. Math. 6 (1995), no. 5, 733-748.

[5] H. Lange and P. E. Newstead, Coherent systems of genus 0, Internat. J. Math. 15 (2004), no. 4, 409-424.

[6] H. Lange and P. E. Newstead, Coherent systems on elliptic curves, Internat. J. Math. 16 (2005), no. 7, 787-805.

[7] J. Le Potier, Faisceaux semi-stable et systèmes cohérents, Vector bundles in Algebraic Geometry, Durham 1993, eds. N. J. Hitchin, P. E. Newstead and W. M. Oxbury, LMS Lecture Notes Series 208, 179-239.

\section{Received: March 1, 2006}

